\title{
Preliminary study on physical activities of preschool children with autism spectrum disorder and perceived health of their parents in Taiwan
}

\author{
Yi Shan Sung1, Yen Ting Lai1, Hsiao Ling Huang2 \\ 1.Department of Physical Medicine and Rehabilitation, National Taiwan University Hospital Hsin-Chu Branch \\ 2.Department of Healthcare Management, Yuanpei University of Medical Technology, Hsinchu, Taiwan
}

\section{Introduction}

Studies revealed that autism spectrum disorder (ASD) children in school age spent less time participating physical activities and participate fewer types of physical activities than children without disabilities. However, there are few researches about physical activities in preschool ASD children. Recent studies show that there are more parental stress and poor perceived health among caregivers of children with ASD. No researches present family factors correlate of ASD children`s participating in physical activities.

Therefore, the aim of our study is to compare the physical activities of ASD preschool children and typical development children and possible relative factors of ASD children at preschool age.

\section{Method}

A total of 28 parents participated in the study: 14 parents of children with ASD diagnosis by children psychiatry doctor, and 14 typical development children (TD) at preschool age. The questionnaire was development by Health Promotion Administration in Taiwan. The parents` self-report questionnaire include children's total physical activities time per week and parents` self-perceived health and stress.

Table 1. Demographic data of two group

\begin{tabular}{|l|c|c|c|}
\hline & ASD $(\mathrm{n}=14)$ & $\mathrm{TD}(\mathrm{n}=14)$ & $P$-value \\
\hline Age (months) & 64.64 & 59.64 & 0.43 \\
\hline Gender(Male) & $14(100 \%)$ & $14(100 \%)$ & \\
\hline family income & - & - & 0.413 \\
\hline
\end{tabular}

ASD:Autism Spectrum Disorder. TD: Typical Development.

\section{Procedure and data analysis}

Ethical clearance was obtained from relevant hospital ethics committees. After signed informed consent was obtained, all parents were asked to filled out the questionnaire.

Independent $t$ test, Chi-square test and spearman-correlation coefficient test were used to compare the results between ASD and TD groups.

\section{Acknowledgements}

We thank the staff of Biotechnology R\&D Center, National Taiwan University Hospital Hsin-Chu Branch for their assistance in statistical analysis.

\section{Result}

28 aged 3-6 years old children participated in this study. The demographic data was presented in Table 1 . There was no significant difference in age, sex and family income between two groups.

Although, there are no significant difference in physical activity time $(P=.22)$ and frequency $(P=.73)$ between ASD children and TD children. But we found that ASD children have less time in physical activities than TD children in one day. ASD children are low-frequency in doing out-of-school physical activity than TD children per week. There are 9 ASD children did not do physical activities out-of- school. Nevertheless, the major correlative factors of ASD children's physical activities were the maternal stress of social activities $(r h o=-.68, p=0.01)$, time pressure $(r h o=$. $71, p=0.00)$ and psychological pressure $(r h o=.58, p=0.03)$.

Table2 Chi-square test of physical Activities

\begin{tabular}{|l|l|l|}
\hline & $\mathrm{X}^{2}$ & $\begin{array}{l}P \text {-value of } \\
\text { Chi-square }\end{array}$ \\
\hline Physical Activity time everyday & 4.4 & 0.22 \\
\hline $\begin{array}{l}\text { Frequency of out-of-school } \\
\text { Physical activity }\end{array}$ & 3.6 & 0.73 \\
\hline
\end{tabular}

Table 3 Correlations of children's physical activity time and maternal pressure

\begin{tabular}{|l|l|}
\hline & $\operatorname{ASD}(\mathrm{n}=14)$ \\
\hline time pressure & $0.71 * *$ \\
\hline psychological pressure & $0.58^{*}$ \\
\hline social activities & $-0.68^{*}$ \\
\hline$* \mathrm{P}<0.01, * * \mathrm{P}<0.001$ &
\end{tabular}

\section{Discussion and Conclusion}

Children with ASD engaged the less time of physical activities with TD children. Due to small sample size, there is no significant difference. The correlation findings of this study provide an evidence that the pressure of mother are relevant to the physical activity time with ASD children. We need to care about the maternal stress and increasing physical activity time of ASD children. 